\title{
Thyroid Tuberculosis Mimicking Carcinoma: case report
}

\author{
S Ait Abderahmane ,M Bensalah, N.Aribi,L Brakni, S.Ouldkablia, Z Kemali
}

Central Hospital of Army, Algiers, Algeria.

Objectives:

\section{Methods:}

\section{Results:}

Thyroid tuberculosis is a rare extra-pulmonary localization, described for the first time by Lebert in 1862 , its frequency is estimated between $0.1-1 \%$ of all locations of tuberculosis. It is defined by the presence of the bacillus of Koch or the existence of specific histological lesions in the thyroid tissue. This condition can have different clinical forms which reflects the diagnostic difficulty. Tuberculosis of the thyroid gland occurs rarely, and should be considered in the diagnosis of nodular lesion of the thyroid gland. The aim of our observation is to report a new case of this location Thyroid Tuberculosis Mimicking Carcinoma and discuss the diagnostic and therapeutic procedures.

We report the case of a 44 year-old woman admitted for therapeutic management of a suspected goiter appeared at 02 months of hospitalization and quickly increasing in volume in a few days, she had a goiter Clinically type II, firm, homogeneous, without palpable nodules in it, hardly moving with swallowing movements with slight dysphagia without signs of thyroid dysfunction.

Cervical Ultrasound: found a suspicious mass in the right thyroid lodge.

FNA of cervical mass found the CPE side for a very granulomatous thyroiditis De Quervain

IDR A TUBERCULIN had regained energy.

The patient received a total thyroidectomy before the signs of malignancy on ultrasound. Histology was in favor of a histopathology of thyroid and thyroid localization soft tissue died a Caseo follicular mise tuberculosis TB treatment with good clinical course.

Thyroid localization of tuberculosis is rare even in endemic areas.

All age groups are affected, the average age is between 30 and 46 years.

The achievement, like the rest of thyroid diseases, interested women rather than men.

. Some pathological situations play a significant role in the development of TT. The latter seems to be favored by advanced age, diabetes, malnutrition and AIDS.

Clinically, there are few signs that guide or ask the diagnosticd'autant more than the TT can take all aspects of thyroid disease. We can meet compression-type signs of dysphagia or paralysis recurrent.

General symptoms may be absent. At the beginning of evolution, hyperthyroidism may occur consecutively to the destruction of the parenchyma and massive release of thyroid hormones. Subsequently, hypothyroidism can occur by total destruction of the gland.

The case of our patient illustrates the difficulty of diagnosis based on simple clinical data: multinodular goiter volume increasing rapidly in less than a week without accompanying clinical signs, infiltrating surrounding tissue with ultrasound, which we had facing a cancer etiology including anaplastic thyroid carcinoma.

Place of thyroid ultrasound is paramount in this diagnostic approach. It allows to give the characteristics of the nodule and thyroid parenchyma adjacent (isolated nodule or nodular goiter) and learn about the presence or absence of lymph nodes.

This is the FNA who will decide the diagnosis by demonstrating the infection by the existence of a épithéliogiganto-cell granuloma with caseous necrosis. Search by BK direct examination or even after culture medium Loewenstein may be unsuccessful.

Thyroid tuberculosis is a rare disease, which can take various forms, especially you must think in TB endemic area, the diagnosis is histological and / or bacteriological. Treatment is primarily medical, based on a combination of anti tuberculosis. Preoperative diagnosis of thyroid tuberculosis is important because of the availability of medical treatments and the limited role of surgery. 